\title{
Unzipped and Defective Nanotubes: Rolling up Graphene and Unrolling Tubes
}

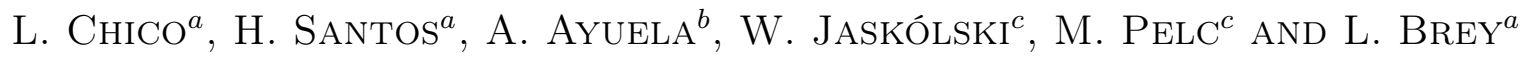 \\ ${ }^{a}$ Instituto de Ciencia de Materiales de Madrid, CSIC, Cantoblanco, 28049 Madrid, Spain \\ ${ }^{b}$ Centro de Física de Materiales CPM CSIC-UPV/EHU, Departamento de Física de Materiales \\ (Facultad de Químicas), and Donostia International Physics Center (DIPC), 20080 Donostia, Spain \\ ${ }^{c}$ Instytut Fizyki UMK, Grudziądzka 5, 87-100 Toruń, Poland
}

\begin{abstract}
The properties of carbon nanotubes can be dramatically altered by the presence of defects. In this work we address the properties of two different kinds of defective nanotubes: junctions of achiral tubes with topological defects and partially unzipped carbon nanotubes. In particular, we begin by focussing on the interface states in carbon nanotube junctions between achiral tubes. We show that their number and energies can be derived by applying the Born-von Karman boundary condition to an interface between armchair- and zigzag-terminated semi-infinite graphene layers. We show that these interface states, which were thought to be due to the presence of topological defects, are in fact related to the graphene zigzag edge states. Secondly, we study partially unzipped carbon nanotubes, which can be considered as the junction of a carbon nanotube and a graphene nanoribbon, which has edge features giving rise to novel properties. Carbon nanoribbons act as transparent contacts for nanotubes and viceversa, yielding a high conductance. At certain energies, nanoribbons behave as valley filters for carbon nanotubes; this holds considering electron-electron interaction effects. Furthermore, the application of a magnetic field turns the system conducting, with a $100 \%$ magnetoresistance. These novel structures may open a way for new carbon-based devices.
\end{abstract}

PACS numbers: 81.05.U-, 73.20.-r, 73.63.Fg, 85.75.-d

\section{Introduction}

Carbon-based materials are presently considered as potential candidates for the development of new nanoelectronic devices, due to their exceptional electronic properties. Nanopatterned graphene, carbon nanotubes (CNT) and graphene nanoribbons (GNR) have been proposed as ingredients in nanocarbon circuits. In these low-dimensional structures, the presence of defects dramatically modifies their electronic properties. This can be done to tune the electronic behavior or to introduce the spin degree of freedom as the relevant transport property.

In this work we focus on two ways of modifying the carbon nanotube electronic structure and transport behavior. Firstly we address the electronic properties of carbon nanotubes with topological defects, such as pentagons and heptagons. By changing the nanotube helicity, its electronic character can be modified, turning a metallic tube into semiconducting or vice versa. Alternatively, topological pentagon-heptagon pair can be regarded as arising from the junction of nanotubes of different chiralities, thus constituting the interfaces between distinct nanotubes, or intramolecular junctions [1]. We pay attention here to the interface states arising in such intramolecular junctions composed of topological defects, and we link its origin to graphene edge states [2].

Secondly, we turn to partially unzipped carbon nanotubes, a novel class of defective tubes that present an open section that can be considered as a carbon nanorib- bon. In such structures, graphene edges dominate the transport properties, rendering the system magnetoresistive. Furthermore, the ribbon section behaves as a valley filter with respect to the carbon nanotube, opening a new way to device design [3].

\section{Model and method}

All the systems studied lack translational symmetry, so we employ a Green function matching technique to calculate the local density of states and quantum conductance in carbon nanotube and graphene junctions. The details are given in Ref. [4].

We employ the most common notation to label the nanotubes, namely, denoting each nanotube by the numbers $(n, m)$, given by the unrolled circumference vector $\boldsymbol{C}_{h}=n \boldsymbol{a}_{1}+m \boldsymbol{a}_{2}$, where $\boldsymbol{a}_{1}, \boldsymbol{a}_{2}$ are the two lattice vectors of graphene at $60^{\circ}$. With this convention, armchair nanotubes have $(n, n)$ indices, and zigzag tubes belong to the $(n, 0)$ series. In particular, a zigzag nanotube with $n$ hexagons around the circumference is denoted by $(n, 0)$.

We have performed the systematic study of achiral nanotube junctions made by joining an armchair $(n, n)$ nanotube to a zigzag $(2 n, 0)$ one. To this end, we have employed the $\pi$-electron tight-binding approximation*.

\footnotetext{
* With one $\pi$ orbital per atom, the hopping parameter for nearest neighbors is fixed to $V_{p p \pi}=-2.66 \mathrm{eV}$.
} 
We have recently shown that for multiple junctions, like $N(12,0) / M(6,6)$ superlattices, this approximation yields the electronic structure around the Fermi energy $\left(E_{\mathrm{F}}\right)$ in good agreement with the results from first-principles calculations [5]. We have checked that the number of interface states given with the tight-binding approach agrees with that obtained with an $a b$ initio approach for the smaller cases, up to $n=7$. The first-principles calculations were carried out using the generalized gradient approximation within density-functional theory [6] employing the SIESTA ab initio method [7]. In order to use the supercell approach, we have calculated the superlattices corresponding to the junctions of interest, thus periodically repeating the interfaces. The calculated cases are $8(6,0) / 8(3,3), 4(8,0) / 4(4,4), 4(12,0) / 4(6,6)$, and $4(14,0) / 4(7,7)$, where the prefactor $N$ in $N(n, m)$ indicates the number of unit cells employed for the superlattices.

For the unzipped nanotubes, besides the non-interacting case, we have included the electronic repulsion within the Hubbard model, which we solve in the mean field approximation. For the employed $\pi$-electron hopping $t \approx 3 \mathrm{eV}$, a Hubbard term in the range $1.5 \mathrm{eV}$ $<U<3 \mathrm{eV}$ describes correctly the main features found in $a b$ initio calculations $[8,9]$ around the Fermi energy. This Hamiltonian reads

$$
H=-t \sum_{i, j, \sigma} c_{i, \sigma}^{\dagger} c_{j, \sigma}+U \sum_{i, \sigma} n_{i, \sigma}\left\langle n_{i,-\sigma}\right\rangle,
$$

where $c_{i, \sigma}^{\dagger}\left(c_{j, \sigma}\right)$ is a creation (annihilation) operator at atom $i(j)$ of a $\pi$ electron with spin $\sigma$ and $n_{i, \sigma}$ are occupation numbers. We model the unzipped part by setting to zero the hopping between the carbon atoms where the opening occurs, and we assume that the unzipping does not affect the hopping parameter between the other carbon atoms, which we set to $t=2.66 \mathrm{eV}$. As this Hamiltonian depends on the electronic occupation, it has to be solved selfconsistently [10].

\section{Interface states in achiral carbon nanotube junctions}

The theoretical proposal of carbon nanotube intramolecular junctions was made quite some time ago, motivated by the possibility of having nanotubes with semiconducting or metallic character. In fact, the potential applications of carbon nanotubes for electronics rely on controlling the synthesis of intramolecular junctions. In the last few years, there have been important advances towards the obtention of such nanotube junctions: Yao et al. reported the synthesis of several carbon nanotube junctions produced by temperature changes during the growth process [11], whereas Jin et al. have reported the formation of nanotube junctions by current injection between tubes [12]. Nanotube junctions are achieved by the presence of topological defects, which allow for the connections of tubes with different chiralities. In many instances, such junctions present interface states, which have been customarily attributed to the presence of the aforementioned topological defects, affecting dramatically the transport properties. In fact, transport spectroscopy measurements have evidenced that interface states have a crucial role in the CNT junction properties [13-15].

The spatial localization of interface states has been experimentally probed. Different decay lengths have been measured in metal/metal and semiconductor junctions: Ishigami et al. studied metal/metal CNT junctions with scanning tunneling microscopy, showing that interface states extend approximately $2 \mathrm{~nm}$ from the junction [14]. On the other hand, Kim et al. found that interface states have a greater spatial extension in semiconductor nanotube junctions, with different values at each side of the interface [15]. Thus, understanding the physics of CNT intramolecular junctions, for which interface states may control transport properties, is an important issue, which has been in fact the focus of increasing interest lately $[4,16-19]$.

In this work, we first describe interface states in achiral nanotube junctions and explain their appearance and origin by studying junctions of varying diameter. Specifically, we address the energy spectra of zigzag/armchair junctions, made by joining a $(2 n, 0)$ and an $(n, n)$ tube. These junctions are achieved by a full ring of $n$ pentagonheptagon pairs. Figure 1 shows an instance of this kind of junctions, the $(10,0) /(5,5)$ case. A full ring of 5 pentagon-heptagon pair defects $(5 / 7)$ joins the two different tubes.

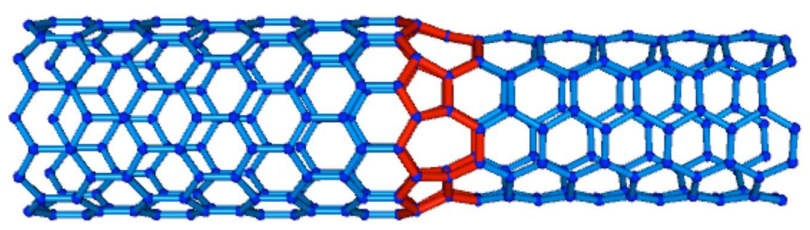

Fig. 1. Geometry of a $(10,0) /(5,5)$ junction. The atoms forming the pentagon-heptagon defects are highlighted in a different color.

Figure 2 shows the local density of states (LDOS) around $E_{\mathrm{F}}$, for all the $(2 n, 0) /(n, n)$ systems from $n=4$ to $n=15$, evaluated at the interface between the nanotubes. Smaller junctions, such as the $(6,0) /(3,3)$ case (not shown), have no localized states, despite the fact that they also have a full ring of $n 5 / 7$ topological defects at the junction. It is evident from Fig. 2 that the appearance of interface states (IS) obeys a multiple-of-three rule: when $n=3 q+1$, with $q=1,2, \ldots$ a new interface state appears, $q$ being the number of such states. Besides, IS can be labeled by an integer number $m$, characterizing the behavior of the wave function $\Psi_{\text {IS }}$ under rotations $C_{n}$ of an angle $\phi=2 \pi / n$. As the junction is invariant under $C_{n}$, it can be seen that $C_{n} \Psi_{\text {IS }}=\mathrm{e}^{\mathrm{i} m \phi} \Psi_{\mathrm{IS}}$. In Fig. 2 a dashed line joins interface states of equal $m$. The index $m$ can be considered as a "discrete angular momentum" quantum number [4]. The interface state energies have a size dependence which recalls that of localized 
states in a quantum well with increasing number of layers [20]. However, in contradistinction to quantum well states, the interface peaks cross with increasing system size. Another particular feature of IS is that their energies are limited to a narrow interval below $E_{\mathrm{F}}$, namely between -0.3 and $0 \mathrm{eV}$.

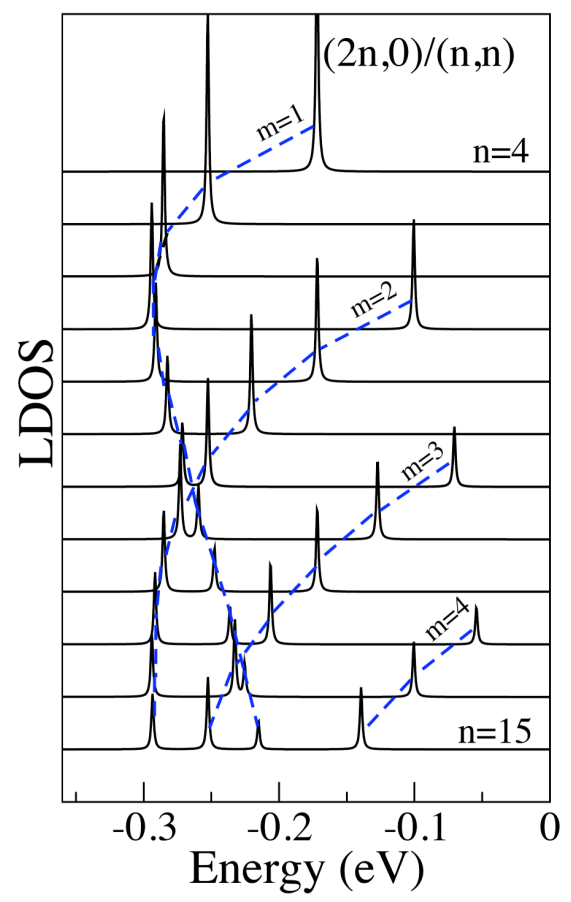

Fig. 2. Local density of states below the Fermi level as a function of system size $n$ for a series of $(2 n, 0) /(n, n)$ junctions. Peaks correspond to interface states. The curves are arranged from top to bottom in order of increasing $n$, with the smallest and largest values indicated therein. Dashed lines are guides to the eye joining states with equal $m$.

Figure 2 also shows that some interface states of junctions with different $n$ have exactly the same energies. For example, the $(10,0) /(5,5)$ and the $(20,0) /(10,10)$ junctions have one IS at the same energy, $-0.2525 \mathrm{eV}$. Also, the $(8,0) /(4,4)$, the $(16,0) /(8,8)$ and the $(24,0) /(12,12)$ junctions have one interface state at $-0.172 \mathrm{eV}$.

The coincidence in energies for some interface states for junctions with a common symmetry and the multiple-of-three rule that governs their appearance suggest that they may have a folding origin. Motivated by these facts, we have considered a system closely related to this series of nanotube junctions: a semi-infinite zigzag graphene joined to an armchair-terminated one by an infinite line of pentagon-heptagon topological defects. This defect line is an interface made by connecting the two graphene edges, as shown in Fig. 3. In the same fashion that a perfect nanotube can be made by rolling up a graphene sheet, an armchair/zigzag carbon nanotube junction as those described above can be obtained by rolling up a strip of the matched semi-infinite graphenes.

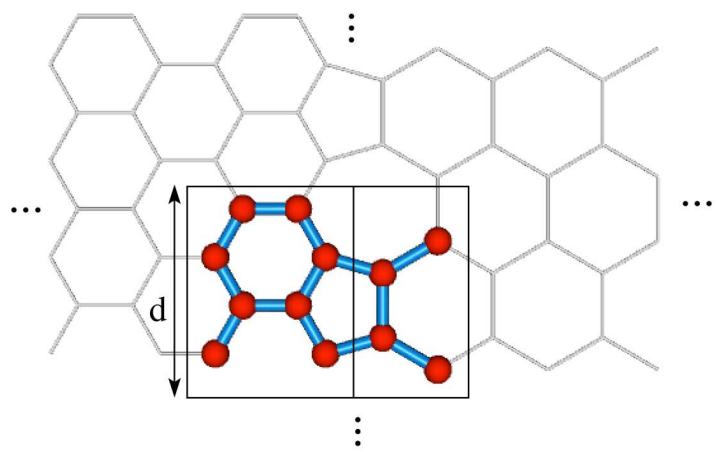

Fig. 3. Geometry of the zigzag/armchair graphene junction. The unit cells employed in the Green function matching calculation are highlighted by two rectangles.

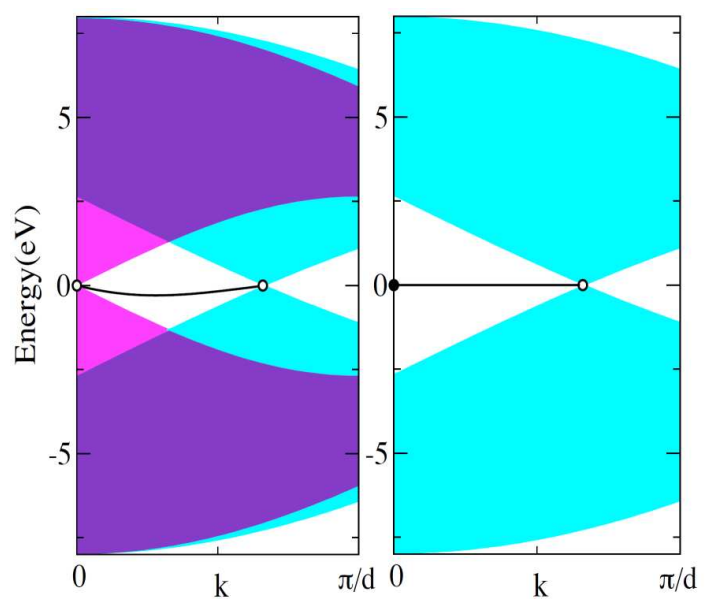

Fig. 4. Left part: interface band of the zigzag/armchair graphene junction, with the corresponding projected graphene bulk bands at this interface. Right part: edge band of the zigzag-terminated graphene with the graphene band structure projected at this surface.

The left part of Fig. 4 depicts the band structure of the armchair graphene/zigzag graphene interface, as well as the projected bulk bands at this interface. The right part shows the edge band of zigzag-terminated graphene with the corresponding projected graphene bulk band structure at this surface. The unit cell employed for this calculation is the same as the one employed for the armchairzigzag graphene junction, in order to make a direct comparison between both results. The interface band shown in the left part spans from $\Gamma$ to $2 / 3$ of the positive part of the Brillouin zone. Just at the edge points there are no interface states, because they belong to the bulk of the armchair- and the zigzag-terminated graphene, respectively. The graphene interface band spans from $-0.3 \mathrm{eV}$ to $0 \mathrm{eV}$, comprising the energy range of all the nanotube interface states. Rolling up the graphene junction is equivalent to imposing Born-von Karman boundary condition to the graphene interface band. This settles 
the quantization rule

$$
k=\frac{2 \pi m}{n d}, \quad m=0, \ldots, n-1,
$$

where $d$ is the length of the repeat unit cell along the interface and $n$ is the number of repetitions to give a $(2 n, 0) /(n, n)$ junction. The index $m$ is the "discrete angular momentum" formerly introduced. The $k$ values given by Eq. (2) yield the nanotube interface states shown in Fig. 2. This is demonstrated graphically in Fig. 5. The energies obtained by this rule exactly match those obtained in the nanotube junction calculations, collected in Fig. 2. The multiple-of-three periodicity is thus under- stood, due to the length of the BZ portion in which the interface graphene band exists, i.e., $2 / 3$ of its irreducible part. Furthermore, within the model employed, it is now clear why for $n<4$ there are no interface states in the $(2 n, 0) /(n, n)$ junctions: in these cases, quantization lines touch the edges of the graphene interface band, and these end points do not actually belong to it because they are in the zigzag and armchair graphene bulk continua. Finally, the appearance of interface states with exactly the same energies is simply explained by the quantization rule given in Eq. (2).
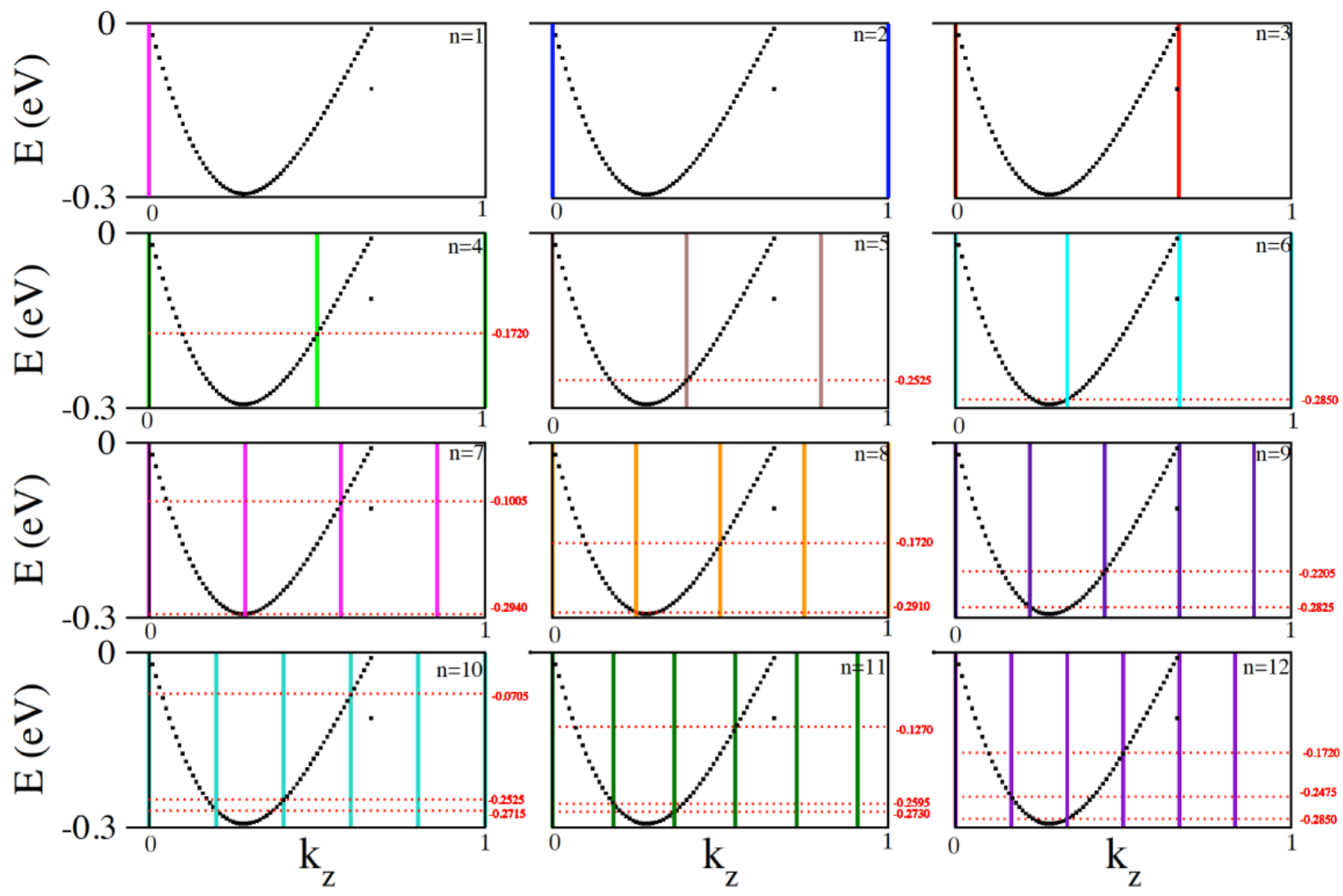

Fig. 5. Zoom of the graphene interface band showing the quantization lines from $n=4$ to $n=12$.

In order to comprehend the origin of the zigzag/ armchair graphene interface band, we have examined the corresponding graphene free "surfaces", the armchair-terminated and the zigzag-terminated semi-infinite graphenes [21]. There are no surface bands in the gap of the armchair-terminated graphene, but for the zigzag-terminated one, a flat band at $0 \mathrm{eV}$ spans from $\Gamma$ to $2 / 3$ of the irreducible part of the BZ, as can be seen in the right part of Fig. 4 . We have used here the same unit cell as for the interface calculation, which is doubled with respect to the one usually employed for zigzag geometry, in order to make a direct comparison with the interface band shown in the left part. Let us note that for the semi- -infinite zigzag graphene, the $k=0$ state belongs to the surface band, because this point is in the bulk gap. This explains why all semi-infinite $(2 n, 0)$ zigzag nanotubes have a "surface" edge state at $0 \mathrm{eV}$. Joining the zigzag edge graphene to the armchair one breaks the electronhole symmetry due to the mixing of the two graphene sublattices. This allows for the dispersion of the surface band, moving it to negative energies, as depicted in the left part of Fig. 4. In this sense, the armchair-edge graphene can be viewed as a finite external potential barrier for the states of the zigzag-terminated graphene, allowing for the penetration of the zigzag edge state wave function into the armchair side and thus bending down 
the interface band. Therefore, we have demonstrated here the zigzag edge nature of the interface band shown in Fig. 4, as well as that of interface states in zigzag/ armchair junctions of tubes. We have seen that they originate from edge states, as those found in graphene nanoribbons. This finding has implications for the analysis of other defects such as vacancies, and even substitutional atoms in nanotubes or graphene, which have been shown to yield an effective edge in the hexagonal carbon lattice [22].

In order to test the validity of our tight-binding results, we have carried out $a b$ initio calculations of $4(2 n, 0) / 4(n, n)$ superlattices (SL) using the same method and parameters as in Ref. [5]. Introducing another junction and imposing periodic boundary conditions induces significant changes in the electronic structure, but by comparison to tight-binding results and checking the wave-function symmetry and spatial distribution, we have successfully identified interface states [23]. Specifically, we have checked that there are no interface states in the $(6,0) /(3,3)$ system, whereas one IS per junction appears in the $(8,0) /(4,4)$ and the $(12,0) /(6,6)$ cases, and two states per junction appear in the $(14,0) /(7,7)$ system. Up to this point, ab initio calculations and tight-binding results fully agree as to the number of interface states in these achiral junctions. For the time being, calculations for superlattices of larger size are beyond our computational capabilities.

We have chosen a pair of interface states belonging to the largest system calculated by ab initio techniques, the $4(14,0) / 4(7,7)$ SL, to show their spatial distribution. Their wave functions are shown in Fig. 6a. The lowest-lying interface state, labeled I1, is mainly localized at the interface, spreading towards the armchair side. This behavior was also observed in the interface states of $(12,0) /(6,6)$ SLs and $(10,0) /(5,5)$ junctions [23, 24]. However, the second interface state (labeled I2) spreads considerably from the interface into the zigzag part. To understand these dissimilar behaviors, we consider again the graphene junction. Figure $6 \mathrm{~b}$ depicts the electron density of several graphene interface states with different $k$ values. The states are labeled with the corresponding $k$ value in $\pi / d$ units. When $k$ goes from $\Gamma$ to the interface band edge at $2 / 3$ of the $\mathrm{BZ}$, the wave-function localization changes from the armchair to the zigzag side. In particular, for $k$ at the band minimum the wavefunction is mainly localized at the junction. This explains why the interface state of the $(12,0) /(6,6)$ junction, which stems from the graphene $k=1 / 3$ state, is rather localized at the interface, and the fact that different states in the same junction may have unlike localizations. In particular, junctions with sufficiently large diameter will have different interface states spreading at opposite sides of the interface, but pinned at the carbon ring made of $5 / 7$ topological defects.

Our results provide an alternative explanation to the dissimilar decay lengths found in semiconductor nanotube junctions, as well as to their large value compared (a)

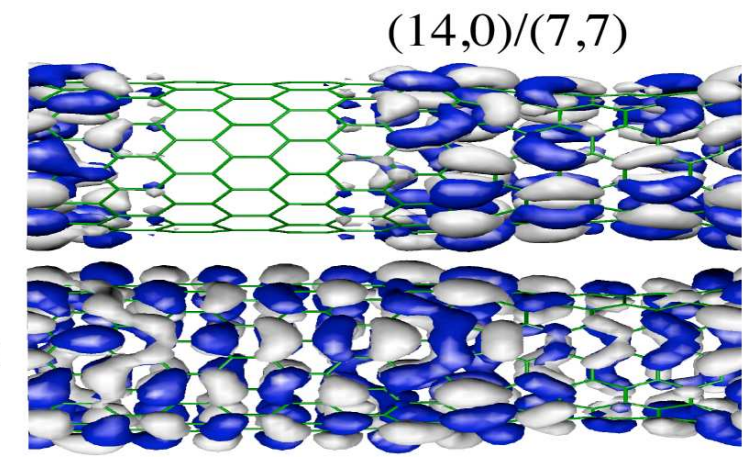

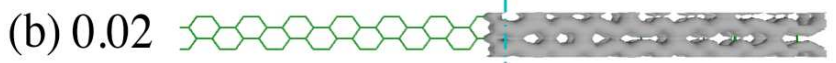

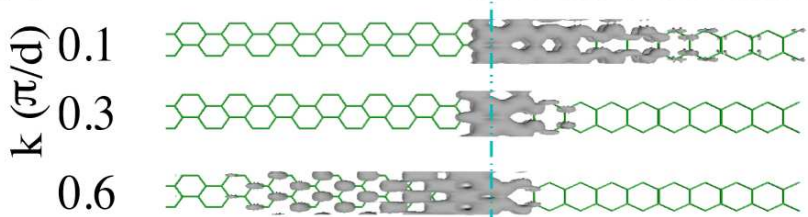

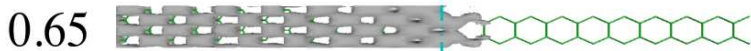

Fig. 6. (a) Two examples of wave functions of interface states belonging to the $4(14,0) / 4(7,7)$ superlattice calculated $a b$ initio. (b) Electron density of several states belonging to the interface band of the graphene armchair/zigzag junction, calculated with a $\pi$-orbital tight-binding model.

to metallic systems $[14,15]$. The coexistence in the same nanotube of interface states with dissimilar spatial localization could be demonstrated by scanning tunneling microscopy and spectroscopy, as in Refs. [13-15]. The fact that CNT junctions may have several interface states with different spatial localizations opens a way for new device design based on their characteristics. Choosing a CNT of appropriate diameter, states with quite different spatial localization can be accessed by applying different voltages, allowing for switch operation.

We have focused on junctions between achiral tubes and found that their interface states have zigzag edge origin, but we would like to note that differences in chirality of joined tubes certainly plays a role. For example, a zigzag $(8,0) /(7,1)$ junction has no interface states, while the $(8,0) /(5,3)$ junction has two [16]. We have chosen to study junctions between tubes with maximum difference in chiral angles. The role of chirality deserves further exploration; but in any case, our present results suggest that IS in chiral systems will also have edge origin.

\section{Partially unzipped nanotubes}

Now we turn to another kind of defective carbon system, the partially unzipped carbon nanotube. The edges produced by the partial unzipping can be considered as defective regions for the nanotube. Two possible geometries of partially unzipped tubes are shown in Fig. 7.

We propose that partial unzipping of carbon nanotubes can actually be used to produce a new class of carbon- 


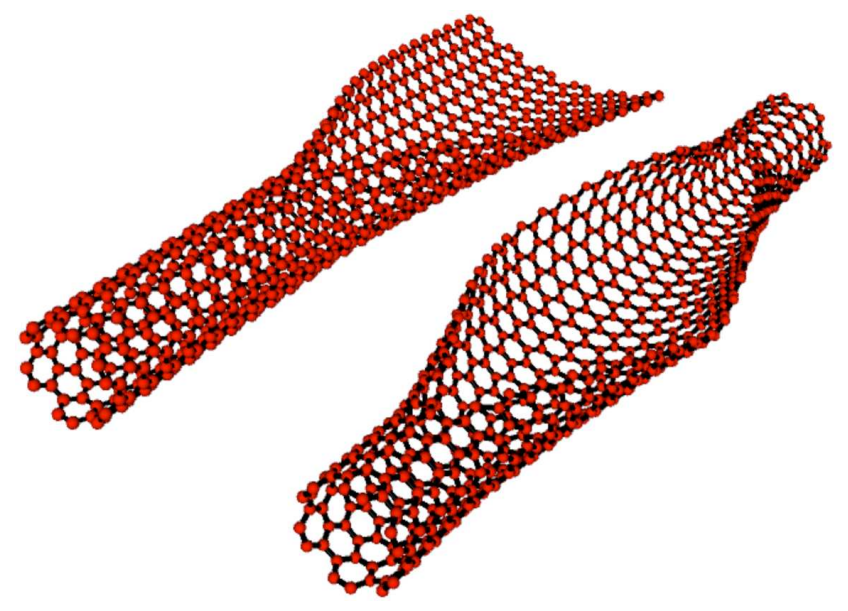

Fig. 7. Geometry of two partially unzipped nanotubes. Left: a $(6,6)$ armchair nanotube unzipped into a 12-ZGNR, making a CNT/GNR single junction. Right: the same nanotube unzipped in its central part, yielding a zigzag nanoribbon quantum dot connected to armchair nanotube contacts.

-based nanostructures, which combine nanoribbons and nanotubes. By studying the GNR/CNT junction we conclude that nanoribbons and nanotubes behave as ideal contacts for each other. Furthermore, we obtain that structures formed by zigzag-terminated GNR and armchair CNT units behave as spin and valley filters, and can be used as building blocks for carbon-based devices featuring very large magnetoresistance.

After the experimental isolation of graphene [25] and the measurement of the anomalous electronic properties of its carriers [26, 27] has increased the theoretical and experimental investigation of its transport properties [28]. The valence and conduction bands of graphene touch at two inequivalent points of the Brillouin zone. Near these points the dispersion relation is linear, so graphene carriers behave as massless Dirac fermions. Due to the large separation in reciprocal space of the Dirac points, intervalley scattering is suppressed in pure graphene samples [29]. Therefore, besides the spin and charge degree of freedom, graphene carriers should be also characterized by a valley index $[28,30]$.

Graphene can be patterned using high-resolution lithography [31], so in principle nanocircuits with transistors and interconnects can be fabricated in the same graphene layer. Narrow (nanometer size) stripes of graphene, called nanoribbons, could be used as quasi-one dimensional connectors [32]. Several groups have worked in the experimental synthesis of graphene nanoribbons. Lithographic techniques have allowed for the production of wide $(>20 \mathrm{~nm})$ stripes of graphene [33, 34], but with limited smoothness. Chemical methods [35-37] have been employed successfully, albeit producing microscopic quantities of graphene nanoribbons. Chemical vapor deposition methods yield macroscopic quantities of nanoribbons, but the samples had a wide dispersion in size and number of layers [38], so the controlled fabrication of nanoribbons of small width remained as a technological challenge.

However, quite recently, three experimental groups announced independently a new promising way to fabricate narrow GNR using CNT as starting material [39-41]. These three groups propose to longitudinally unzip CNTs to make nanoribbons, either by chemical attack [39], by plasma etching [40], or by lithium intercalation followed by exfoliation [41], with very high yields. Unzipping carbon nanotubes appears as a promising way to fabricate narrow nanoribbons with smooth edges, as needed for nanoelectronic applications.

From the theoretical viewpoint, carbon nanoribbons are obtained by cutting graphene in the form of a quasi-one-dimensional stripe. The electronic properties of GNR strongly depend on the atomic edge termination. There are two basic shapes for graphene edges, armchair and zigzag [42]. The GNR electronic properties can be derived from the graphene band structure by imposing the appropriate boundary conditions [43]. Armchair GNR can be either metallic or semiconducting depending of their width, whereas for the zigzag GNR (ZGNR) twofold degenerated flat bands lie at the Fermi energy. These bands are associated with edge states [43] and their dispersionless character favors an insulating antiferromagnetic ground state, with opposite magnetization at the edges [9, 42, 44-46]. Zigzag graphene nanoribbons are obtained by unrolling an armchair CNT, see Fig. 7. The ZGNR width is defined by the number $n$ of zigzag rows in the unit cell; the usual notation for such ribbon is $n$-ZGNR. We study here partially unzipped carbon nanotubes, such as those shown in Fig. 7, which are equivalent to a combination of carbon nanotubes and graphene nanoribbons. In what follows, we focus in armchair carbon nanotubes and the derived ZGNR.

In Fig. 8 we show the conductance of a single $(6,6)$ CNT/12-ZGNR junction, as the one depicted in the upper part of Fig. 7, for noninteracting electrons $(U=0)$. As a reference, we show in dashed and dotted lines the conductances of the perfect infinite CNT and ZGNR. Around zero energy, the conductance of the junction is equal to that of the perfect nanoribbon, demonstrating that the $(6,6) \mathrm{CNT}$ acts as a transparent contact for the 12-ZGNR. Assuming left to right conduction, the ZGNR only has the $K^{\prime}$ channel open to transport and it is completely transparent to states from the corresponding $K^{\prime}$ valley of the CNT. This indicates that backscattering is practically zero in the device and the conductance in this energy range is set by the ribbon, which acts as a valley filter for the CNT. Our numerical results show that states from the $K$ valley of the CNT cannot transverse the junction. At higher energies, more channels open in the ribbon, so the conductance now is limited by that of the $(6,6)$ nanotube: here, the ribbon is acting as a transparent contact for the CNT, and transport from both valleys is allowed. Calculations with larger systems, such as a $(18,18) \mathrm{CNT} / 36-Z \mathrm{GNR}$ (not shown here) yield similar 
results, with the obvious increase in the number of channels. We have also investigated the narrowing of the ribbon part, such as a $(6,6) \mathrm{CNT} / 10-Z \mathrm{GNR}$, or a $(18,18) /$ $32-Z G N R$, finding that the effect is robust against the precise form of the $\mathrm{CNT} / \mathrm{ZGNR}$ junction.

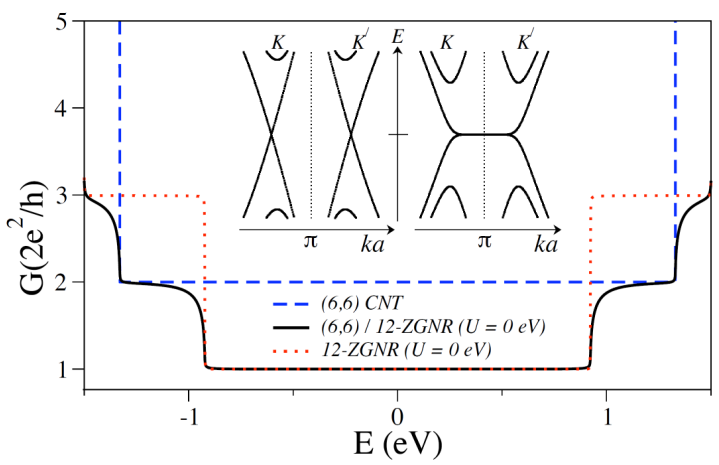

Fig. 8. Solid line: conductance of a $(6,6) \mathrm{CNT} / 12$ -ZGNR junction. Dashed (dotted) line: conductance of a perfect infinite $(6,6) \mathrm{CNT}(12-\mathrm{ZGNR})$. The results correspond to $U=0$. The insets show the band structures of the armchair nanotube (left) and the zigzag nanoribbon (right). The flat bands joining the Dirac points in the ZGNR correspond to states localized at the edges of the ribbon.

As discussed above, the interaction between electrons changes dramatically the band structure of graphene nanoribbons near the Fermi energy. Figure 9 shows the effect of electron-electron interactions in the conductance of the $(6,6) \mathrm{CNT} / 12-\mathrm{ZGNR}$ junction for $U=2 \mathrm{eV}$ and $U=3 \mathrm{eV}$. The inset shows the bandstructure for the $(6,6) \mathrm{CNT}$ and for the 12-ZGNR for an interaction $U=3 \mathrm{eV}$. For CNTs the Hubbard interaction does not modify the magnetic moments on the carbon atoms, so the effect of $U$ is just a rigid shift of the electronic structure. In the case of ZGNR the interaction orders ferromagnetically the most external atoms at each edge, and the magnetic moments on opposite edges couple antiferromagnetically. Magnetic order induces dispersion of the edge bands along the edge direction, opening a gap at the center of the band structure that increases with the value of the interaction $U$. The states with opposite spin orientation are degenerated, but the edge bands with opposite spin correspond to states located at opposite edges. The many body-induced gap in ZGNR precludes transport near the Fermi energy. Above the gap, there is a region of enhanced conductance with respect to the non-interacting case. This occurs because the dispersion of the edge states, induced by the electron-electron interaction, opens a new electronic channel near each Dirac point of the ZGNR. This is the case at point $C$ in the inset of Fig. 9. Besides transmission from $K^{\prime}$ to $K^{\prime}$ valleys observed in the non-interacting case, now a state $A$ in the CNT $K$ valley can be transmitted into state $C$ of the ZGNR at the same valley, giving an enhanced conductance. The width of this bump in the conductance is proportional to the midband gap and increases with $U$.
The state $C$ is an edge state and for opposite spin orientations the wave function of this state is localized in opposite edges. Therefore the excess of current with respect to the noninteracting case is localized at the edges and with opposite spin polarization. Above this energy region there is an energy interval where the conductance gets the value $2 e^{2} / h$, and valley filtering occurs, as for the non-interacting case. This energy interval is around $0.6 \mathrm{eV}$ for $U=2 \mathrm{eV}$, so the observation of this filtering could be observed in carbon nanotubes. Above this interval, the conductance values are quite similar to the non-interacting cases, demonstrating the high transparency of armchair CNTs for ZGNR and vice versa.

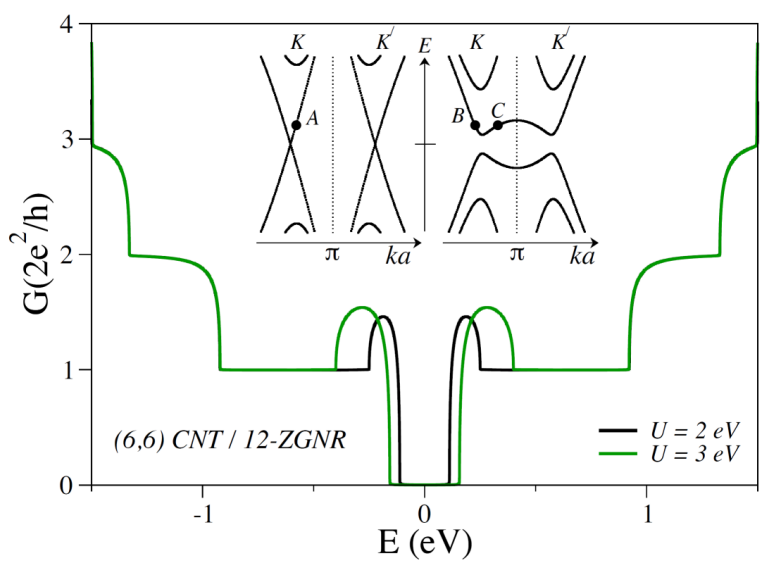

Fig. 9. Conductance of a $(6,6) \mathrm{CNT} / 12$ ZGNR with on-site repulsion $(U=2$ and $3 \mathrm{eV})$. Insets show the band structures of the armchair nanotube (left) and the zigzag nanoribbon (right) for $U=3 \mathrm{eV}$.

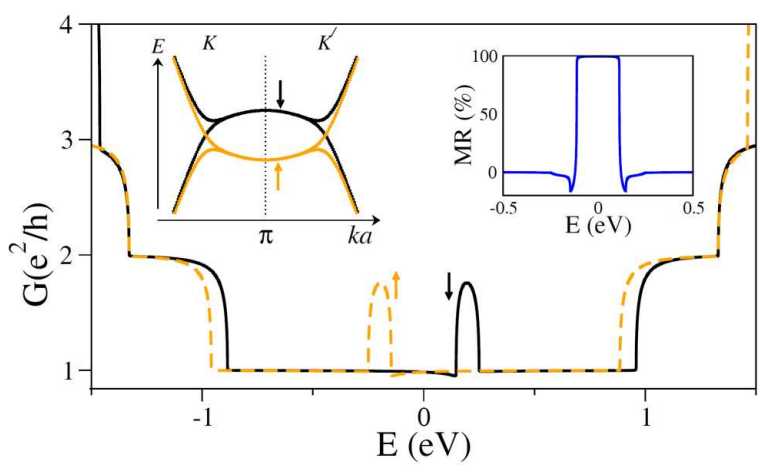

Fig. 10. Conductance of a $(6,6) \mathrm{CNT} / 12$ ZGNR with on-site repulsion $(U=2 \mathrm{eV})$ in the ferromagnetic configuration. Left inset: band structure of the ferromagnetic zigzag nanoribbon. Dark (light) lines correspond to the spin orientation parallel (antiparallel) to the applied magnetic field. Right inset: magnetoresistance of the device as a function of energy.

The application of a magnetic field makes the ZGNR ferromagnetic and metallic [46], as can be seen in Fig. 10, which presents the conductance of a $(6,6)$ nanotube/12- 


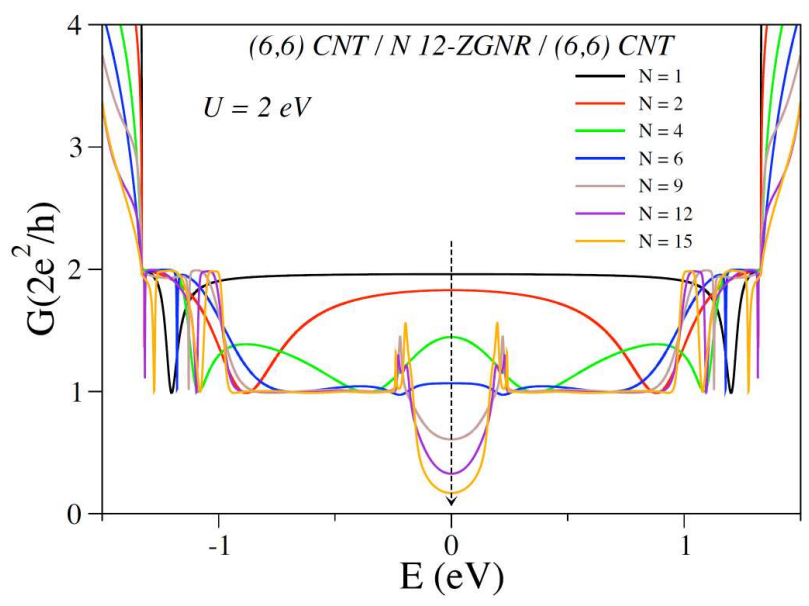

Fig. 11. Conductance of a $(6,6) \mathrm{CNT} / N$ 12-ZGNR/ $(6,6) \mathrm{CNT}$ with on-site repulsion $U=2 \mathrm{eV}$ for several ribbon lengths $(N=1$ to $N=15)$. The arrow indicates increasing system size.

-ZGNR junction with the edges ferromagnetically coupled. The magnetic field closes the midgap, as shown in the left inset, opening new channels at low energies. This gives rise to large magnetoresistance around the Fermi energy, as depicted in the right inset of Fig. 10. We propose that a single unzipped carbon nanotube is by itself a device featuring $100 \%$ magnetoresistance.

We have also explored the properties of double junction systems, such as the one depicted in Fig. 7 (right), an infinite armchair $(6,6)$ nanotube open in its central part making a ZGNR. We denote this structure as $(6,6)$ $\mathrm{CNT} / N 12$-ZGNR/(6,6) $\mathrm{CNT}$, where $N$ is the number of unit cells in the nanoribbon. The transparency of the nanotube contacts is evident in Fig. 11: the transmission through the central ribbon part is higher that in an infinite ribbon for the smaller sizes, and slowly decays to the zero limit value in the gap with increasing ribbon size. Other combinations, such a CNT quantum dot with ribbon contacts can be envisioned, expanding the possibilities of carbon electronics, in analogy to the quantum dot and superlattice structures proposed for CNTs [18, 19].

\section{Conclusions}

In summary, we have explored two types of defective nanotubes. First we have focused in carbon nanotube junctions made of achiral tubes, which may present interface states. We have shown that these states, usually attributed to the pentagon-heptagon topological defects which constitute the junction, are in fact due to the zigzag-edge-terminated nanotube. Topological defects break the electron symmetry and consequently make these states energy-dependent. Furthermore, we have related these interface states of nanotube junctions to the interface band appearing in a graphene zigzag/armchair junction. By applying the Born-von Karman boundary condition to the graphene interface band, we have derived the energies and number of the nanotube interface states, obtaining complete quantitative agreement with the CNT junction calculations. Our results give a new vision on the nature of CNT interface states and have implications in other systems, such as graphene vacancies or substitutional impurities.

Secondly, we have studied novel carbon nanostructures based on unzipped nanotubes. These structures have defective edges, which are responsible for their interesting transport properties which actually consist of mixed carbon nanotube/nanoribbon systems. We have found that ribbons from unzipped tubes behave as completely transparent contacts for the parent tubes, and vice versa. Our results demonstrate that partially unzipped carbon nanotubes are by themselves magnetoresistive devices, with a large value of the magnetoresistance. Furthermore, carbon nanoribbons act as valley filters for carbon nanotubes; this behavior is robust with respect to the inclusion of electron-electron interaction. This opens the possibility of exploiting the valley degree of freedom in a new class of carbon-based nanodevices.

\section{Acknowledgments}

L.C. acknowledges helpful discussions with J.I. Cerdá. This work has been partially supported by the Spanish DGES under grants MAT2006-06242, MAT2006-03741 and FIS2007-66711-C02-C01 and Spanish CSIC under grant PI 200860I048. W.J. and M.P. acknowledge financial support from Polish LFPPI.

\section{References}

[1] D. Wei, Y. Liu, Adv. Mater. 20, 2815 (2008).

[2] H. Santos, A. Ayuela, W. Jaskólski, M. Pelc, L. Chico, Phys. Rev. B 80, 035436 (2009).

[3] H. Santos, L. Chico, L. Brey, Phys. Rev. Lett. 103, 086801 (2009).

[4] L. Chico, L.X. Benedict, S.G. Louie, M.L. Cohen, Phys. Rev. B 54, 2600 (1996).

[5] A. Ayuela, L. Chico, W. Jaskólski, Phys. Rev. B 77, 085435 (2008).

[6] J.P. Perdew, K. Burke, M. Ernzerhof, Phys. Rev. Lett. 77, 3865 (1996).

[7] J.M. Soler, E. Artacho, J.D. Gale, A. García, J. Junquera, P. Ordejón, D. Sánchez-Portal, J. Phys., Condens. Matter 14, 2745 (2002).

[8] J. Fernández-Rossier, J.J. Palacios, Phys. Rev. Lett. 99, 177204 (2007).

[9] J. Fernández-Rossier, Phys. Rev. B 77, 075430 (2008).

[10] M.P. López Sancho, M.C. Muñoz, L. Chico, Phys. Rev. B 63, 165419 (2001).

[11] Y. Yao, Q. Li, J. Zhang, R. Liu, L. Jiao, Y.T. Zhu, Z. Liu, Nature Mater. 6, 283 (2007).

[12] C. Jin, K. Suenaga, S. Iijima, Nature Nanotech. 3, 17 (2008). 
[13] H. Kim, J. Lee, S.-J. Kahng, Y.-W. Son, S.B. Lee, C.-K. Lee, J. Ihm, Y. Kuk, Phys. Rev. Lett. 90, 216107 (2003).

[14] M. Ishigami, H.J. Choi, S. Aloni, S.G. Louie, M.L. Cohen, A. Zettl, Phys. Rev. Lett. 93, 196803 (2004).

[15] H. Kim, J. Lee, S. Lee, Y. Kuk, J.-Y. Park, S.-J. Kahng, Phys. Rev. B 71, 235402 (2005).

[16] L. Chico, V.H. Crespi, L.X. Benedict, S.G. Louie, M.L. Cohen, Phys. Rev. Lett. 76, 971 (1996).

[17] R. Saito, G. Dresselhaus, M.S. Dresselhaus, Phys. Rev. B 53, 2044 (1996); J.C. Charlier, Ph. Lambin, T.W. Ebbesen, Phys. Rev. B 53, 11108 (1996).

[18] L. Chico, M.P. López Sancho, M.C. Muñoz, Phys. Rev. Lett. 81, 1278 (1998); C.G. Rocha, T.G. Dargam, A. Latgé, Phys. Rev. B 65, 165431 (2002); W. Zhang, W. Lu, E.G. Wang, Phys. Rev. B 72, 075438 (2005); F. Triozon, P. Lambin, S. Roche, Nanotechnology 16230 (2005); E. Jódar, A. Pérez-Garrido, A. Díaz-Sánchez, Phys. Rev. B 73, 205403 (2006).

[19] L. Chico, W. Jaskólski, Phys. Rev. B 69, 085406 (2004); W. Jaskólski, L. Chico, Phys. Rev. B 71, 155305 (2005).

[20] J.E. Ortega, F.J. Himpsel, Phys. Rev. Lett. 69 844 (1992); A. Ayuela, E. Ogando, N. Zabala, Phys. Rev. B 75, 153403 (2007).

[21] M. Fujita, K. Wakabayashi, K. Nakada, K. Kusakabe, J. Phys. Soc. Jpn. 65, 1920 (1996); K. Wakabayashi, M. Fujita, A. Ajiki, M. Sigrist, Phys. Rev. B 59, 8271 (1999).

[22] Y. Gan, L. Sun, F. Banhart, Small 4, 587 (2008).

[23] A. Ayuela, W. Jaskolski, M. Pelc, H. Santos, L. Chico, Appl. Phys. Lett. 93, 133106 (2008).

[24] A. Rochefort, Ph. Avouris, Nano Lett. 2, 253 (2002).

[25] K.S. Novoselov, A.K. Geim, S.V. Mozorov, D. Jiang, Y. Zhang, S.V. Dubonos, I.V. Gregorieva, A.A. Firsov, Science 306, 666 (2004).

[26] K.S. Novoselov, D. Jiang, T. Booth, V. Khotkevich, S.M. Morozov, A.K. Geim, Nature 438, 197 (2005).

[27] Y. Zhang, Y.-W. Tan, H.L. Störmer, P. Kim, Nature 438, 201 (2005).

[28] A.H. Castro-Neto, F. Guinea, N.M.R. Peres, K.S. Novoselov, A.K. Geim, Rev. Mod. Phys. 81, 109 (2009).

[29] A.F. Morpurgo, F. Guinea, Phys. Rev. Lett. 97, 196804 (2006).

[30] A. Rycerz, J. Tworzydlo, C.W.J. Beenakker, Nature Phys. 3, 172 (2007).
[31] C. Berger, Z. Song, X. Li, X. Wu, N. Brown, C. Naud, D. Mayou, T. Li, J. Hass, A.N. Marchenkov, E.H. Conrad, P.N. First, W.A. de Heer, Science $\mathbf{3 1 2}$ 1191 (2006).

[32] A. Iyengar, T. Luo, H.A. Fertig, L. Brey, Phys. Rev. B 78, 235411 (2008).

[33] M.Y. Han, B. Ozyilmaz, Y. Zhang, P. Kim, Phys. Rev. Lett. 98, 206805 (2007).

[34] B. Özyilmaz, P. Jarillo-Herrero, D. Efetov, D.A. Abanin, L.S. Levitov, P. Kim, Phys. Rev Lett. 99, 166804 (2007).

[35] H.C. Schniepp, J.-L. Li, M.J. McAllister, H. Sai, M. Herrera-Alonso, D.H. Adamson, R.K. Prud'homme, R. Car, D.A. Saville, I.A. Aksay, J. Phys. Chem. B 110, 8535 (2006).

[36] X. Li, X. Wang, L. Zhang, S. Lee, H. Dai, Science 319, 1229 (2008).

[37] X. Yang, X. Dou, A. Rouhanipour, L. Zhi, H.J. Rader, K. Mullen, J. Am. Chem. Soc. 130, 4126 (2008).

[38] J. Campos-Delgado, J.M. Romo-Herrera, X. Jia, D.A. Cullen, H. Muramatsu, Y.A. Kim, T. Hayashi, Z. Ren, D.J. Smith, Y. Okuno, T. Ohba, H. Kanoh, K. Kaneko, M. Endo, H. Terrones, M.S. Dresselhaus, M. Terrones, Nano Lett. 8, 2773 (2008).

[39] D. Kosynkin, A.-L. Higginbotham, A. Sinitskii, J.R. Lomeda, A. Dimiev, B.K. Price, J.M. Tour, $\mathrm{Na}$ ture 458, 872 (2009).

[40] L. Jiao, L. Zhang, X. Wang, G. Diankov, H. Dai, Nature 458, 877 (2009).

[41] A.G. Cano-Márquez, F.J. Rodríguez-Macías, J. Campos-Delgado, C.G. Espinosa-González, F. Tristán-López, D. Ramírez-González, D.A. Cullen, D.J. Smith, M. Terrones, Y.I. Vega-Cantú, Nano Lett. 9, 1527 (2009).

[42] M. Fujita, K. Wakabayashi, K. Nakada, K. Kusakabe, J. Phys. Soc. Jpn. 65, 1920 (1996).

[43] L. Brey, H. Fertig, Phys. Rev. B 73, 325411 (2006).

[44] Y.W. Son, M.L. Cohen, S.G. Louie, Phys. Rev. Lett. 97, 216803 (2006).

[45] L. Pisani, J. Chan, B. Montanari, N. Harrison, Phys. Rev. B 75, 064418 (2007).

[46] F. Muñoz-Rojas, J. Fernández-Rossier, J.J. Palacios, Phys. Rev. Lett. 102, 136810 (2009). 